PROCEEDINGS OF THE

AMERICAN MATHEMATICAL SOCIETY

Volume 138, Number 6, June 2010, Pages 2051-2060

S 0002-9939(09)10207-1

Article electronically published on December 8, 2009

\title{
ON PLURI-HALF-ANTICANONICAL SYSTEMS OF LEBRUN TWISTOR SPACES
}

\author{
NOBUHIRO HONDA
}

(Communicated by Jon G. Wolfson)

\begin{abstract}
In this paper, we investigate pluri-half-anticanonical systems on the so-called LeBrun twistor spaces. We determine its dimension, the base locus, the structure of the associated rational map, and also the structure of general members, in precise form. In particular, we show that if $n \geq 3$ and $m \geq 2$, the base locus of the system $\left|m K^{-1 / 2}\right|$ on $n \mathbb{C P}^{2}$ consists of two nonsingular rational curves, along which any member has singularity, and that if we blow up these curves, then the strict transform of a general member of $\left|m K^{-1 / 2}\right|$ becomes an irreducible non-singular surface. We also show that if $n \geq 4$ and $m \geq n-1$, then the last surface is a minimal surface of general type with vanishing irregularity. We also show that the rational map associated to the system $\left|m K^{-1 / 2}\right|$ is birational if and only if $m \geq n-1$.
\end{abstract}

\section{INTRODUCTION}

Recently a large number of new Moishezon twistor spaces was obtained [2, 3, 4, An important common property of these twistor spaces is that they all admit a $\mathbb{C}^{*}$-action. This implies that the corresponding self-dual metrics admit an isometric $U(1)$-action. Another common property of the twistor spaces is that the linear system $\left|K^{-1 / 2}\right|$ (so called the fundamental system) is a pencil. Actually, the structure of the twistor spaces was investigated by making use of reducible members of this pencil. It is also remarkable that no example is known so far of a Moishezon twistor space whose $\left|K^{-1 / 2}\right|$ is empty (or even 0-dimensional). The author does not know at all whether such a twistor space exists or not, but it is certain that, if it exists, it has to be studied through the linear system $\left|m K^{-1 / 2}\right|$ for some $m \geq 2$, the pluri-half-anticanonical systems.

With this background, the purpose of this paper is to give detailed results on the structure of the pluri-half-anticanonical systems for the most fundamental Moishezon twistor spaces, the LeBrun twistor spaces [6, §7]. It turns out that it is rather easy to express the dimension of the system $\left|m K^{-1 / 2}\right|$ as a function of $n$ and $m$ in completely explicit form (Proposition 2.2). The dimension formula immediately implies that, for LeBrun twistor spaces on $n \mathbb{C P}^{2}$, any element of $\left|m K^{-1 / 2}\right|$ with $m<n-1$, is a pull-back of a curve of bidegree $(m, m)$ on $\mathbb{C P}^{1} \times \mathbb{C P}^{1}$ under the

Received by the editors June 29, 2009, and, in revised form, September 7, 2009, and September $15,2009$.

2010 Mathematics Subject Classification. Primary 32L25; Secondary 53C28.

The author was partially supported by the Grant-in-Aid for Young Scientists (B), The Ministry of Education, Culture, Sports, Science and Technology, Japan.

(C)2009 American Mathematical Society Reverts to public domain 28 years from publication 
rational map associated to $\left|K^{-1 / 2}\right|$. It readily follows from this that any element of $\left|m K^{-1 / 2}\right|$ with $m<n-1$ has ordinary singularity of multiplicity $m$ along two rational curves which are the base curves of $\left|K^{-1 / 2}\right|$ (Corollary 2.3). The formula also implies that if $m \geq n-1$, general members of $\left|m K^{-1 / 2}\right|$ are not a pull-back of any curve on $\mathbb{C P}^{1} \times \mathbb{C P}^{1}$. However, we can show that even such members always have a singularity along the same rational curves (Proposition 2.4, which includes more detailed information on the structure of $\left.\left|m K^{-1 / 2}\right|\right)$. In particular, they are nonnormal. This is in contrast with a result shown by Pedersen-Poon [7, Lemma 2.1], which means that any real irreducible members of $\left|K^{-1 / 2}\right|$ are smooth in general. Also, noting that the line bundle $K^{-1 / 2}$ is big for any Moishezon twistor space, this singularity result indicates some peculiar property of LeBrun twistor spaces as compact complex manifolds. We also determine precisely the multiplicity of general members of $\left|m K^{-1 / 2}\right|$ along the base curves (Proposition 2.4 (ii)), and also show that if we blow up the two base curves, then the divisor becomes an irreducible, non-singular surface (Proposition 2.4 (iii)). Further, we determine the structure of the last non-singular surface (Proposition 2.8). Finally, we investigate the structure of the rational map associated to $\left|m K^{-1 / 2}\right|$. Thus principal information on the pluri-half-anticanonical system of LeBrun twistor spaces is fully obtained.

Notation. For line bundles $L$ and $L^{\prime}$, we often write $L+L^{\prime}$ for the tensor product $L \otimes L^{\prime}$, and $L^{m}$ or $m L$ for $L^{\otimes m}$. We write $h^{i}(L)$ for $\operatorname{dim} H^{i}(L)$. The notation $\operatorname{dim}|L|$ means $h^{0}(L)-1$ as usual. For a complex submanifold $X$ in a complex manifold $Y, N_{X / Y}$ denotes the normal bundle of $X$ in $Y . K$ means the canonical bundle. The symbol $\sim$ denotes a linear equivalence.

\section{StUdy ON PLURI-HALF-ANTICANONICAL SYSTEMS}

Fix an arbitrary integer $n \geq 3$ and let $Z$ be any LeBrun twistor space on $n \mathbb{C P}^{2}$, constructed in [6, §7]. We do not put any assumption for the dimension of the automorphism group for $Z$, so that the corresponding self-dual metric (the LeBrun metric) on $n \mathbb{C P}^{2}$ may admit a 2-torus action or just a $U(1)$-action. Let $F$ be the canonical half of the anticanonical line bundle on $Z$, and let $\sigma$ be the real structure on $Z$. Recall that the degree of a divisor or a line bundle on $Z$ is defined as the intersection number with a twistor line. Then $\operatorname{deg} F=2$, and among all line bundles on $Z$ of positive degree, $F$ can be characterized by the reality (namely $\bar{\sigma}^{*} F \simeq F$ ) and minimality of the degree.

The structure of the complete linear system $|F|$ has been completely understood by the works of LeBrun [6], Poon [9] and Kurke [5].

Proposition 2.1. Let $Z$ and $F$ be as above. Then we have:

(i) $\operatorname{dim}|F|=3$,

(ii) $\operatorname{Bs}|F|$ consists of two non-singular rational curves which are mutually disjoint and conjugate,

(iii) a general member of $|F|$ is a non-singular rational surface, which is obtained from $\mathbb{C P}^{1} \times \mathbb{C P}^{1}$ by blowing up $2 n$ points.

Let $C_{1}$ and $\bar{C}_{1}=\sigma\left(C_{1}\right)$ be the base curves of $|F|$. (These curves will play an important role in the following computation.) Let $\Phi: Z \rightarrow \mathbb{C P}^{3}$ be the rational map associated to the system $|F|$, so that its indeterminacy locus is $C_{1} \cup \bar{C}_{1}$. Then basically by the existence of two (mutually conjugate) pencils whose degree is one, by which $|F|$ is generated, the image of the rational map $\Phi$ is a non-singular 
quadratic surface. Hence the image $\Phi(Z)$ is isomorphic to $\Sigma_{0}:=\mathbb{C P}^{1} \times \mathbb{C P}^{1}$. Let $\mu: \tilde{Z} \rightarrow Z$ be the blowing-up along $C_{1} \cup \bar{C}_{1}$, and let $E_{1}$ and $\bar{E}_{1}$ be the exceptional divisors over $C_{1}$ and $\bar{C}_{1}$, respectively. Then the composition $\tilde{\Phi}:=\Phi \circ \mu$ becomes a morphism (onto $\Sigma_{0}$ ), and $E_{1}$ and $\bar{E}_{1}$ become sections of $\tilde{\Phi}$. By the properties $N_{C_{1} / Z} \simeq \mathscr{O}(1-n)^{\oplus 2}$ and $N_{\bar{C}_{1} / Z} \simeq \mathscr{O}(1-n)^{\oplus 2}$ and an important property that the fiber directions of $\left.\mu\right|_{E_{1}}: E_{1} \rightarrow C_{1}$ and $\left.\mu\right|_{\bar{E}_{1}}: \bar{E}_{1} \rightarrow \bar{C}_{1}$ are different with respect to the isomorphism $E_{1} \simeq \bar{E}_{1}$ obtained from $\tilde{\Phi}$, we have [6, page 246]

$$
N_{1}:=N_{E_{1} / \tilde{Z}} \simeq \mathscr{O}(1-n,-1), \quad \bar{N}_{1}:=N_{\bar{E}_{1} / \tilde{Z}} \simeq \mathscr{O}(-1,1-n) .
$$

(So $\mathscr{O}_{E_{1}}(1,0)$ and $\mathscr{O}_{\bar{E}_{1}}(0,1)$ are supposed to be the fiber classes of $\left.\mu\right|_{E_{1}}$ and $\left.\mu\right|_{E_{1}}$ respectively.) Thus the situation is summarized in the following basic diagram:

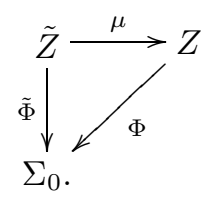

Then as $\left.\mathscr{O}(1)\right|_{\Sigma_{0}} \simeq \mathscr{O}(1,1)$, by the above situation, we have the basic relation

$$
\mu^{*} F \simeq \tilde{\Phi}^{*} \mathscr{O}_{\Sigma_{0}}(1,1)+E_{1}+\bar{E}_{1} .
$$

We first compute the dimension of $H^{0}\left(F^{m}\right)$.

Proposition 2.2. For any $m \geq 1$, we have the natural isomorphism

$$
\begin{aligned}
& H^{0}\left(Z, F^{m}\right) \simeq H^{0}\left(\Sigma_{0}, \mathscr{O}(m, m)\right) \\
& \oplus\left(\bigoplus_{1 \leq k \leq \frac{m}{n-1}} H^{0}\left(\Sigma_{0}, \mathscr{O}(m-k(n-1), m-k) \oplus \mathscr{O}(m-k, m-k(n-1))\right)\right) .
\end{aligned}
$$

In particular we have

$$
H^{0}\left(Z, F^{m}\right) \simeq H^{0}\left(\Sigma_{0}, \mathscr{O}(m, m)\right), \quad m<n-1 .
$$

Proof. By the Leray spectral sequence for the blowup $\mu$, we readily obtain

$$
H^{i}\left(Z, F^{m}\right) \simeq H^{i}\left(\tilde{Z}, \mu^{*} F^{m}\right), \quad \forall i \geq 0 .
$$

To compute the right-hand side, we first notice that by (2.3)

$$
\begin{aligned}
R^{q} \tilde{\Phi}_{*}\left(\mu^{*} F^{m}\right) & \simeq R^{q} \tilde{\Phi}_{*}\left(\tilde{\Phi}^{*} \mathscr{O}(m, m)+m E_{1}+m \bar{E}_{1}\right) \\
& \simeq \mathscr{O}(m, m) \otimes R^{q} \tilde{\Phi}_{*} \mathscr{O}\left(m E_{1}+m \bar{E}_{1}\right)
\end{aligned}
$$

Further, as fibers of $\tilde{\Phi}$ are at most a string of smooth rational curves, we have

$$
R^{q} \tilde{\Phi}_{*} \mathscr{O}\left(m E_{1}+m \bar{E}_{1}\right)=0, \quad \forall q>0, \forall m \geq 0 .
$$

Hence the spectral sequence

$$
E_{2}^{p, q}=H^{p}\left(\Sigma_{0}, R^{q} \tilde{\Phi}_{*}\left(\mu^{*} F^{m}\right)\right) \Rightarrow H^{p+q}\left(\tilde{Z}, \mu^{*} F^{m}\right)
$$

degenerates at the $E_{2}$-term, and we obtain that

$$
H^{i}\left(\tilde{Z}, \mu^{*} F^{m}\right) \simeq H^{i}\left(\Sigma_{0}, \mathscr{O}(m, m) \otimes \tilde{\Phi}_{*} \mathscr{O}\left(m E_{1}+m \bar{E}_{1}\right)\right), \quad \forall i \geq 0 .
$$

For the right-hand side, by taking the direct image of an obvious exact sequence,

$$
0 \longrightarrow \mathscr{O}\left((k-1)\left(E_{1}+\bar{E}_{1}\right)\right) \longrightarrow \mathscr{O}\left(k\left(E_{1}+\bar{E}_{1}\right)\right) \longrightarrow N_{1}^{k} \oplus \bar{N}_{1}^{k} \longrightarrow 0,
$$


and computing $\operatorname{Ext}^{1}\left(\simeq H^{1}\right)$ by using (2.1), we inductively obtain isomorphisms

$$
\tilde{\Phi}_{*} \mathscr{O}\left(m\left(E_{1}+\bar{E}_{1}\right)\right) \simeq \mathscr{O} \oplus\left(\bigoplus_{1 \leq k \leq m}\left(N_{1}^{k} \oplus \bar{N}_{1}^{k}\right)\right), \quad \forall m \geq 0,
$$

where on the right-hand side we are identifying $E_{1}$ and $\bar{E}_{1}$ with $\Sigma_{0}$ by the morphism $\tilde{\Phi}$. Therefore by (2.6) and (2.8) we have

$$
H^{i}\left(Z, F^{m}\right) \simeq H^{i}\left(\Sigma_{0}, \mathscr{O}(m, m)\right) \oplus\left(\bigoplus_{k=1}^{m} H^{i}\left(\Sigma_{0}, N_{1}^{k}(m, m) \oplus \bar{N}_{1}^{k}(m, m)\right)\right), \quad \forall i \geq 0,
$$

where $N_{1}^{k}(m, m)$ means $N_{1}^{k} \otimes \mathscr{O}(m, m)$, and the same for $\bar{N}_{1}^{k}(m, m)$. By (2.1) we have

$$
N_{1}^{k}(m, m) \simeq \mathscr{O}(m-k(n-1), m-k), \quad \bar{N}_{1}^{k}(m, m) \simeq \mathscr{O}(m-k, m-k(n-1)) .
$$

In particular, if $m-k(n-1)<0$, then $H^{0}\left(\Sigma_{0}, N_{1}^{k}(m, m) \oplus \bar{N}_{1}^{k}(m, m)\right)=0$. Therefore (2.10) means the desired isomorphism (2.4). In particular if $m /(n-1)<$ 1 , namely, if $m<n-1$, then the second direct summand does not appear and we obtain (2.5). This completes the proof of Proposition 2.2.

We note that from the proof we have the same isomorphism for $H^{1}\left(F^{m}\right)$; it suffices to remove the requirement for $k$ in (2.4).

By (2.3), we have an inclusion $H^{0}\left(\Sigma_{0}, \mathscr{O}(m, m)\right) \subset H^{0}\left(Z, F^{m}\right)$ for any $m \geq 1$ from the beginning. Hence the isomorphism (2.5) implies:

Corollary 2.3. When $1 \leq m \leq n-2$, we have the following:

(i) Any member of the system $\left|F^{m}\right|$ is of the form $\mu\left(\tilde{\Phi}^{-1}(D)\right)$, where $D$ is a curve on $\Sigma_{0}$ whose bidegree is $(m, m)$. In particular, all members are $\mathbb{C}^{*}$-invariant.

(ii) A general member $Y$ of $\left|F^{m}\right|$ is an irreducible non-normal surface which is birational to a ruled surface of genus $(m-1)^{2}$. Further, $Y$ has ordinary singularity of multiplicity $m$ along $C_{1} \cup \bar{C}_{1}$.

The first statement might be more clearly stated by remarking that the system $\left|F^{m}\right|$ is generated by $|F|$ when $m<n-1$; in other words, $H^{0}\left(F^{m}\right)=\operatorname{Sym}^{m} H^{0}(F)$ holds for $m<n-1$. Thus the situation becomes apparent when $m<n-1$. Next we investigate what happens for the alternative case $m \geq n-1$.

Proposition 2.4. Fix any $m \geq n-1$ and put $l:=[m /(n-1)]$ (the biggest integer not greater than $m /(n-1))$. Let $\mathscr{I} \subset \mathscr{O}_{Z}$ be the ideal sheaf of the curve $C_{1} \cup \bar{C}_{1}$. Then we have the following:

(i) There is the following sequence of subspaces of $H^{0}\left(Z, F^{m}\right)$ :

$$
\begin{aligned}
\tilde{\Phi}^{*} H^{0}(\mathscr{O}(m, m)) & \simeq H^{0}\left(F^{m} \otimes \mathscr{I}^{m}\right) \subsetneq H^{0}\left(F^{m} \otimes \mathscr{I}^{m-1}\right) \\
\subsetneq \ldots & \subsetneq H^{0}\left(F^{m} \otimes \mathscr{I}^{m-l+1}\right) \subsetneq H^{0}\left(F^{m} \otimes \mathscr{I}^{m-l}\right)=H^{0}\left(F^{m}\right) .
\end{aligned}
$$

Moreover for the differences of the dimensions, we have

$$
h^{0}\left(F^{m} \otimes \mathscr{I}^{m-k}\right)-h^{0}\left(F^{m} \otimes \mathscr{I}^{m-k+1}\right)=2 h^{0}(\mathscr{O}(m+k(1-n), m-k)) .
$$


(ii) Take any $1 \leq k \leq l$ and let $Y$ be a general member of the system $\mid F^{m} \otimes$ $\mathscr{I}^{m-k} \mid$. Then $Y$ has ordinary singularity of multiplicity $m-k$ along $C_{1} \cup \bar{C}_{1}$, and the strict transform $\tilde{Y}$ of $Y$ into $\tilde{Z}$ is irreducible and non-singular.

Proof. Let $s_{1} \in H^{0}\left(\mathscr{O}_{\tilde{Z}}\left(E_{1}+\bar{E}_{1}\right)\right)$ be an element satisfying $\operatorname{div}\left(s_{1}\right)=E_{1}+\bar{E}_{1}$. Let $1 \leq k \leq l$ and consider the following injection of sheaves on $\tilde{Z}$ :

$$
\mu^{*} F^{m}-(m-k+1)\left(E_{1}+\bar{E}_{1}\right) \stackrel{\otimes s_{7}}{\longrightarrow} \mu^{*} F^{m}-(m-k)\left(E_{1}+\bar{E}_{1}\right) .
$$

Since

$$
\begin{aligned}
\left.\left(\mu^{*} F^{m}-(m-k)\left(E_{1}+\bar{E}_{1}\right)\right)\right|_{E_{1}} & \left.\simeq\left(\tilde{\Phi}^{*} \mathscr{O}(m, m)+k\left(E_{1}+\bar{E}_{1}\right)\right)\right|_{E_{1}} \\
& \simeq \mathscr{O}(m, m)+k \mathscr{O}(1-n,-1) \\
& \simeq \mathscr{O}_{E_{1}}(m+k(1-n), m-k),
\end{aligned}
$$

and an analogous isomorphism holds for the restriction on $\bar{E}_{1}$, we obtain an exact sequence:

$$
\begin{aligned}
0 & \longrightarrow \mu^{*} F^{m}-(m-k+1)\left(E_{1}+\bar{E}_{1}\right) \stackrel{\otimes s_{1}}{\longrightarrow} \mu^{*} F^{m}-(m-k)\left(E_{1}+\bar{E}_{1}\right) \\
& \longrightarrow \mathscr{O}_{E_{1}}(m+k(1-n), m-k) \cup \mathscr{O}_{\bar{E}_{1}}(m-k, m+k(1-n)) \longrightarrow 0 .
\end{aligned}
$$

Let $\mathscr{L}_{k}$ denote the last non-trivial term of (2.15) (supported on $E_{1} \cup \bar{E}_{1}$ ). As for the sign for the integers in $\mathscr{L}_{k}$, we readily have $m+k(1-n) \geq 0$ and $m-k>0$ under the assumptions $m \geq n-1$ and $1 \leq k \leq l$. Therefore we have

$$
H^{1}\left(\mathscr{L}_{k}\right)=0 \quad(1 \leq k \leq l) .
$$

On the other hand, if $k=1$, the first non-trivial term of (2.15) becomes isomorphic to $\tilde{\Phi}^{*} \mathscr{O}(m, m)$ by (2.3), so that its $H^{1}$ also vanishes. Therefore, the cohomology exact sequence for (2.15) in the case $k=1$ gives an exact sequence

$$
0 \longrightarrow H^{0}\left(\tilde{\Phi}^{*} \mathscr{O}(m, m)\right) \stackrel{\otimes s_{1}}{\longrightarrow} H^{0}\left(\mu^{*} F^{m}-(m-1)\left(E_{1}+\bar{E}_{1}\right)\right) \longrightarrow H^{0}\left(\mathscr{L}_{1}\right) \longrightarrow 0
$$

and also the vanishing (from (2.15) with $k=1$ )

$$
H^{1}\left(\mu^{*} F^{m}-(m-1)\left(E_{1}+\bar{E}_{1}\right)\right)=0 .
$$

Therefore by (2.16), we inductively obtain the exact sequence

$$
\begin{aligned}
0 \longrightarrow H^{0}\left(\mu^{*} F^{m}-(m-k+1)\left(E_{1}+\bar{E}_{1}\right)\right) & \stackrel{\otimes s_{1}}{\longrightarrow} H^{0}\left(\mu^{*} F^{m}-(m-k)\left(E_{1}+\bar{E}_{1}\right)\right) \\
& \longrightarrow H^{0}\left(\mathscr{L}_{k}\right) \longrightarrow 0 \quad(1 \leq k \leq l)
\end{aligned}
$$

and the vanishing

$$
H^{1}\left(\mu^{*} F^{m}-(m-k)\left(E_{1}+\bar{E}_{1}\right)\right)=0 \quad(1 \leq k \leq l) .
$$


By using the exact sequences (2.19) successively, we obtain

$$
\begin{aligned}
h^{0}\left(F^{m} \otimes \mathscr{I}^{m-l}\right) & =h^{0}\left(F^{m} \otimes \mathscr{I}^{m-l+1}\right)+h^{0}\left(\mathscr{L}_{l}\right) \\
& =h^{0}\left(F^{m} \otimes \mathscr{I}^{m-l+2}\right)+h^{0}\left(\mathscr{L}_{l-1}\right)+h^{0}\left(\mathscr{L}_{l}\right) \\
& =\cdots \\
& =h^{0}\left(F^{m} \otimes \mathscr{I}^{m}\right)+\sum_{1 \leq k \leq l} h^{0}\left(\mathscr{L}_{k}\right) \\
& =h^{0}(\mathscr{O}(m, m))+\sum_{1 \leq k \leq l} h^{0}\left(\mathscr{L}_{k}\right) .
\end{aligned}
$$

This directly gives the sequence (2.12) and the dimension formula (2.13). Hence we obtain (i).

For the second claim, we first note that, since $|\mathscr{O}(m, m)|$ (on $\Sigma_{0}$ ) is base point free, by the injection in the exact sequence (2.17), we obtain Bs $\mid \mu^{*} F^{m}-(m-1)\left(E_{1}+\right.$ $\left.\bar{E}_{1}\right) \mid \subset E_{1} \cup \bar{E}_{1}$. Further, as $\mathscr{L}_{1}=\mathscr{O}(m+1-n, m-1) \cup \mathscr{O}(m-1, m+1-n)$ and $m+1-n \geq 0$ and $m-1>0$ as already remarked, we have $\operatorname{Bs}\left|\mathscr{L}_{1}\right|=\emptyset$. Therefore the surjectivity of the restriction map in (2.17) implies Bs $\left|\mu^{*} F^{m}-(m-1)\left(E_{1}+\bar{E}_{1}\right)\right|=\emptyset$. Once this is obtained, again by using the exact sequences (2.19) and verifying $\operatorname{Bs}\left|\mathscr{L}_{k}\right|=\emptyset$ for $1 \leq k \leq l$, we inductively obtain

$$
\text { Bs }\left|\mu^{*} F^{m}-(m-k)\left(E_{1}+\bar{E}_{1}\right)\right|=\emptyset, \quad 1 \leq k \leq l .
$$

Therefore by Bertini's theorem, a general member of the system $\mid \mu^{*} F^{m}-(m-$ $k)\left(E_{1}+\bar{E}_{1}\right) \mid$ is irreducible and non-singular (when $\left.1 \leq k \leq l\right)$. Let $\tilde{Y}$ be such a member. Then if we put $Y:=\mu(\tilde{Y})$, since $\left.\tilde{Y}\right|_{E_{1}} \in|\mathscr{O}(m+k(1-n), m-k)|$ and $\left.\tilde{Y}\right|_{E_{1}} \in|\mathscr{O}(m-k, m+k(1-n))|$, taking into account for the directions of the blowing-down $\mu$ (see (2.1) $)$, this gives a general member of the system $\left|F^{m} \otimes \mathscr{I}^{m-k}\right|$, which has ordinary singularity of multiplicity $m-k$ along $C_{1} \cup \bar{C}_{1}$. Thus we obtain the claim of (ii), and we finish a proof of Proposition 2.4.

Remark 2.5. It is possible to give generators of the system $\left|F^{n-1}\right|$ in completely explicit form. They can be chosen in such a way that all irreducible components of the generators are degree 1 divisors. It might be interesting to remark that the situation is almost the same for the twistor spaces studied in [3]; namely, LeBrun twistor spaces and the twistor spaces in [3] have a common property, at least for the linear system $\left|F^{n-1}\right|$. (See [3, Lemma 2.8]; also compare Proposition 2.8 below with [3, Theorem 4.3].)

As an immediate consequence of Proposition 2.4 we obtain

Corollary 2.6. For any LeBrun twistor spaces on $n \mathbb{C P}^{2}$ with $n \geq 3$, a divisor belonging to the system $\left|F^{m}\right|$ can be non-singular only when $m=1$.

Recall that by a result of Pedersen-Poon [7, on any twistor spaces on $n \mathbb{C P}^{2}$, all real irreducible members of the system $|F|$ are non-singular. Corollary 2.6 shows that this is true only for the system $|F|$ itself. We also remark that when $n=2$, Proposition 2.4 and Corollary 2.6 do not hold, since in that case $\mathrm{Bs}|F|=\emptyset([$, Proposition 2.6]).

Remark 2.7. One might think that since the restriction of $F^{m}$ to a non-singular member $S \in|F|$ is isomorphic to $K_{S}^{-m}$, and since any member of $\left|K_{S}^{-m}\right|$ is readily seen to contain the curve $C_{1}$ and $\bar{C}_{1}$ by a high multiplicity, any member of $\left|F^{m}\right|$ 
would have singularity along $C_{1} \cup \bar{C}_{1}$. But of course this argument just shows that the restriction $\left.Y\right|_{S}$ (where $Y \in\left|F^{m}\right|$ ) contains $C_{1} \cup \bar{C}_{1}$ as non-reduced components and does not show that $Y$ itself has singularity along $C_{1} \cup \bar{C}_{1}$.

Next we investigate the structure of the strict transform of a general member of $\left|F^{m}\right|$ in the case $m \geq n-1$. Because the structure of members of the smallest subsystem $\left|F^{m} \otimes \mathscr{I}^{m}\right|$ in the sequence (2.12) is already clear as in Corollary 2.3 (ii), we consider the case $Y \in\left|F^{m} \otimes \mathscr{I}^{m-k}\right|$ with $1 \leq k \leq l$. (Recall that $\left|F^{m} \otimes \mathscr{I}^{m-l}\right|=$ $\left|F^{m}\right|$ by Proposition 2.4 (i).)

Proposition 2.8. Let $Z$ be any LeBrun twistor space on $n \mathbb{C P}^{2}(n \geq 3), m \geq n-1$ an integer, $l=[m /(n-1)], Y$ a general member of the system $\left|F^{m} \otimes \mathscr{I}^{m-k}\right|$ with $1 \leq k \leq l$, and $\tilde{Y}$ the strict transform of $Y$ into $\tilde{Z}$ as before. Then we have:

(i) The restriction $\left.\tilde{\Phi}\right|_{\tilde{Y}}: \tilde{Y} \rightarrow \Sigma_{0}$ is surjective and its degree is $2 k$.

(ii) If $n \geq 4$ or if $n=3$ and $m>2$, then $\tilde{Y}$ is a (non-singular) minimal surface of general type with vanishing irregularity.

(iii) If $n=3$ and $m=2$, then $\tilde{Y}$ is a (non-singular) $K 3$ surface.

Proof. (i) is immediate from $\tilde{Y} \sim \tilde{\Phi}^{*} \mathscr{O}(m, m)+k\left(E_{1}+\bar{E}_{1}\right)$, recalling that $E_{1}$ and $\bar{E}_{1}$ are sections of $\tilde{\Phi}$ and that the image of $\tilde{\Phi}^{*}|\mathscr{O}(m, m)|$ is strictly smaller than $\left|F^{m}\right|$ when $m \geq n-1$ and $1 \leq k \leq l$ by Proposition 2.2 or Proposition 2.4. For (ii) and (iii), by the adjunction formula, we have $\left.K_{\tilde{Y}} \simeq\left(K_{\tilde{Z}}+\tilde{Y}\right)\right|_{\tilde{Y}}$. Further we have

$$
\begin{aligned}
K_{\tilde{Z}}+\tilde{Y} & \simeq \mu^{*} K_{Z}+\left(E_{1}+\bar{E}_{1}\right)+\tilde{Y} \\
& \simeq \mu^{*}\left(F^{-2}\right)+\left(E_{1}+\bar{E}_{1}\right)+\mu^{*} F^{m}-(m-k)\left(E_{1}+\bar{E}_{1}\right) \\
& \simeq \mu^{*}\left(F^{m-2}\right)-(m-k-1)\left(E_{1}+\bar{E}_{1}\right) \\
& \left.\simeq \tilde{\Phi}^{*} \mathscr{O}(m-2, m-2)+(k-1)\left(E_{1}+\bar{E}_{1}\right) \quad \text { (by (2.3) }\right) .
\end{aligned}
$$

Therefore, since we have $m-2>0$ if $n \geq 4$, or $m>2$ if $n=3$, and since $l-1 \geq 0$, we obtain that $h^{0}\left(\nu K_{\tilde{Y}}\right)$ grows quadratically as a function of $\nu$. Hence $\tilde{Y}$ is a surface of general type. On the other hand, if $n=3$ and $m=2$, we have $l=1$ and hence (2.23) means that $K_{\tilde{Y}}$ is trivial. Next we prove that $H^{1}\left(\mathscr{O}_{\tilde{Y}}\right)=0$ for both cases of (ii) and (iii). For this, by the standard exact sequence $0 \longrightarrow \mathscr{O}_{\tilde{Z}}(-\tilde{Y}) \longrightarrow$ $\mathscr{O}_{\tilde{Z}} \longrightarrow \mathscr{O}_{\tilde{Y}} \longrightarrow 0$, it suffices to show that $H^{2}\left(\mathscr{O}_{\tilde{Z}}(-\tilde{Y})\right)=0$. By duality, the last space is the dual of $H^{1}\left(K_{\tilde{Z}}+\tilde{Y}\right)$. Moreover, by $(2.23)$, we obtain, with the aid of (2.9),

$$
H^{1}\left(K_{\tilde{Z}}+\tilde{Y}\right) \simeq H^{1}\left(\mathscr{O}(m-2, m-2) \otimes\left(\mathscr{O} \oplus\left(N_{1} \oplus \bar{N}_{1}\right) \oplus \cdots \oplus\left(N_{1}^{k-1} \oplus \bar{N}_{1}^{k-1}\right)\right) .\right.
$$

As $N_{1} \simeq \mathscr{O}(1-n,-1)$, in order to prove $H^{1}\left(K_{\tilde{Z}}+\tilde{Y}\right)=0$, it is enough to show that, if we write $\mathscr{O}(m-2, m-2) \otimes N_{1}^{k-1} \simeq \mathscr{O}(a, b)$, then $a \geq 0$ and $b \geq 0$ hold. For this, putting $m=l(n-1)+q$ with $0 \leq q<n-1$ as before, we compute

$$
a=m-2+(k-1)(1-n) \geq l(n-1)+q-2+(l-1)(1-n)=n+q-3 \geq 0
$$

and

$$
b=m-k-1 \geq l(n-1)+q-l-1=l(n-2)+q-1 \geq q \geq 0 .
$$

Hence we obtain $H^{1}\left(K_{\tilde{Z}}+\tilde{Y}\right)=0$, so that $H^{1}\left(\mathscr{O}_{\tilde{Y}}\right)=0$. In particular, in the situation of (iii), $\tilde{Y}$ is a $K 3$ surface. 
To complete a proof of Proposition [2.8, it remains to prove that $\tilde{Y}$ does not contain a $(-1)$-curve, in the situation of (ii). Since $\left(\tilde{\Phi}^{*} \mathscr{O}(m-2, m-2)\right) \cdot D \geq 0$ for any curve $D$ on $\tilde{Y}$, by (2.23) we have $K_{\tilde{Y}} \cdot D \geq 0$ for any curve $D \subset \tilde{Y}$ unless $D \subset E_{1} \cup \bar{E}_{1}$. So to prove that there is no $(-1)$-curve, it suffices to show that if we take a sufficiently general $Y$, then the restriction $\left.\tilde{Y}\right|_{E_{1}}$ does not contain a (-1)-curve. For this, by (2.14) we have $\left.\tilde{Y}\right|_{E_{1}} \in|\mathscr{O}(m+k(1-n), m-k)|$. Further, we have

$$
m+k(1-n)=l(n-1)+q+k(1-n)=(l-k)(n-1)+q \geq q
$$

and

$$
m-k=l(n-1)+q-k \geq l(n-1)+q-l=l(n-2)+q \geq 2 .
$$

Therefore (by the surjectivity of the restriction map in the exact sequence (2.19)), if we take sufficiently general $Y \in\left|F^{m} \otimes \mathscr{I}^{m-k}\right|$, the curve $\left.\tilde{Y}\right|_{E_{1}}$ is a non-singular irreducible curve of bidegree $(a, b)$ with $a \geq q$ and $b \geq 2$. Hence if $q>1, a>1$ follows and hence $\left.\tilde{Y}\right|_{E_{1}}$ cannot be a rational curve for sufficiently general $Y$. On the other hand, if $a=0$, we obtain $l=k$ and $q=0$, and hence $\left.\tilde{Y}\right|_{E_{1}}$ becomes a curve of bidegree $(0, l(n-2))$, so that it is a union of $l(n-2)$ smooth rational curves of bidegree $(0,1)$, for sufficiently general $Y$. Now since $\tilde{Y}$ intersects $E_{1}$ transversally along these rational curves, the self-intersection numbers of these curves in $\tilde{Y}$ are

$$
\mathscr{O}(0,1) \cdot N_{E_{1} / \tilde{Z}}=\mathscr{O}(0,1) \cdot \mathscr{O}(1-n,-1)=1-n<-2 .
$$

Therefore any of the $l(n-2)$ rational curves cannot be a $(-1)$ curve on $\tilde{Y}$. Finally, if $a=1$, then $l=k$ and $q=1$ follow and the curve $\left.\tilde{Y}\right|_{E_{1}}$ becomes a non-singular rational curve of bidegree $(1, l(n-2)+1)$. However we have

$$
\mathscr{O}(1, l(n-2)+1) \cdot N_{E_{1} / \tilde{Z}}=-l(n-1)(n-2)-n \leq-1 \cdot 2 \cdot 3-4=-10 .
$$

This means that the self-intersection number of $\left.\tilde{Y}\right|_{E_{1}}$ in $\tilde{Y}$ is less than -9 . Hence it cannot be a (-1)-curve, too. By the same reason, any curve $D \subset \tilde{Y} \cap \bar{E}_{1}$ cannot be a $(-1)$-curve for general $Y \in\left|F \otimes \mathscr{I}^{m-k}\right|$. Thus we have proved that $\tilde{Y}$ does not contain a (-1)-curve. Namely, it is a minimal surface.

It might be interesting to remark that as in the assertion (i) of the proposition, the degree of the map $\left.\tilde{\Phi}\right|_{\tilde{Y}}: \tilde{Y} \rightarrow \Sigma_{0}$ depends on $k$, not on $m$. Also, for another basic invariant of the surface $\tilde{Y}$ of general type, it is possible to compute $K_{\tilde{Y}}^{2}$ for $\tilde{Y}$ in Proposition 2.8 (ii).

Finally we show the following result about the rational map associated to the pluri-half-anticanonical systems:

Proposition 2.9. Let $Z$ be any LeBrun twistor space on $n \mathbb{C P}^{2}$ with $n \geq 3$, and let $\Phi_{m}$ be the rational map associated to the linear system $\left|F^{m}\right|$ on $Z$. Then we have the following.

(i) If $1 \leq m \leq n-2$, the rational map $\Phi_{m}$ factors as $g_{m} \circ \Phi_{1}$, where $g_{m}$ is a holomorphic map from $\Sigma_{0}$ associated to the linear system $|\mathscr{O}(m, m)|$. (In particular, the image $\Phi_{m}(Z)$ is biholomorphic to $\Sigma_{0}$.)

(ii) If $m \geq n-1$, the map $\Phi_{m}$ is birational over its image. 
Proof. (i) is obvious from (2.5) or Corollary 2.3. For (ii), it is enough to show the case $m=n-1$. For this, from the natural commutative diagram

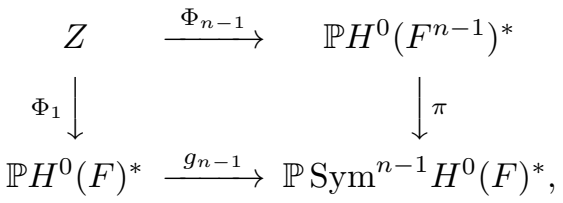

where $\pi$ is the linear projection induced by the inclusion $\operatorname{Sym}^{n-1} H^{0}(F) \subset H^{0}\left(F^{n-1}\right)$ and $g_{n-1}$ is the Veronese embedding associated to the linear system $|\mathscr{O}(n-1)|$ on $\mathbb{P} H^{0}(F)^{*}$, we obtain that the map $\Phi=\Phi_{1}: Z \rightarrow \Sigma_{0}$ factors as

$$
Z \stackrel{\Phi_{n-1}}{\longrightarrow} \Phi_{n-1}(Z) \stackrel{\pi}{\longrightarrow} \Sigma_{0}
$$

(so that we are identifying $\Phi(Z)$ and $g_{n-1}\left(\Sigma_{0}\right)$ by $\left.g_{n-1}\right)$. This means that $\Phi_{n-1}$ does not identify two different fibers of $\Phi$. So in order to show the desired birationality, it suffices to show that, for a general fiber $f$ of $\tilde{\Phi}$ (which is a non-singular rational curve), the restriction of the composition $\left.\left(\Phi_{n-1} \circ \mu\right)\right|_{f}$ is biholomorphic.

To show this, we first note that the composition $\Phi_{n-1} \circ \mu$ is the rational map associated to the linear system $\left|\mu^{*} F^{n-1}\right|$. Further, by Proposition 2.4 (i), this system has $(n-2)\left(E_{1}+\bar{E}_{1}\right)$ as a fixed component since now $l=[m /(n-1)]=1$. Then as the system $\left|\mu^{*} F^{n-1}-(n-2)\left(E_{1}+\bar{E}_{1}\right)\right|$ is base point free by (2.22) (while $m=n-1$ and $l=k=1$ ), we obtain that $\Phi_{n-1} \circ \mu$ is a morphism associated to the free linear system $\left|\mu^{*} F^{n-1}-(n-2)\left(E_{1}+\bar{E}_{1}\right)\right|$. From the relation (2.3) the last linear system is linearly equivalent to $\tilde{\Phi}^{*} \mathscr{O}(n-1, n-1)+\left(E_{1}+\bar{E}_{1}\right)$. The intersection number of this line bundle with the fiber $f$ being 2 , the restriction $\left.\left(\Phi_{n-1} \circ \mu\right)\right|_{f}$ is either $2: 1$ over the image or biholomorphic to the image.

In order to eliminate the former possibility, we recall that $Z$ admits a $\mathbb{C}^{*}$-action, and the center $C_{1} \cup \bar{C}_{1}$ is contained in its fixed locus. Hence the blowup $\tilde{Z}$ still has a $\mathbb{C}^{*}$-action, leaving the exceptional divisors $E_{1}$ and $\bar{E}_{1}$ invariant. Also, any fiber of $\tilde{\Phi}$, and therefore also of $f$, is $\mathbb{C}^{*}$-invariant. In particular, the two distinct intersection points $E_{1} \cap f$ and $\bar{E}_{1} \cap f$ are $\mathbb{C}^{*}$-fixed points. This means that, if $\left.\left(\Phi_{n-1} \circ \mu\right)\right|_{f}$ is $2: 1$ over the image, then the two fixed points have to be the ramification points of the double cover. But this cannot happen, since the system $\tilde{\Phi}^{*} \mathscr{O}(n-1, n-1)+\left(E_{1}+\bar{E}_{1}\right)$ actually has a member of the form $\tilde{\Phi}^{-1}(A)+E_{1}+\bar{E}_{1}$ with $A \in \mathscr{O}(n-1, n-1)$, and hence the two points $E_{1} \cap f$ and $\bar{E}_{1} \cap f$ are identified by $\left.\left(\Phi_{n-1} \circ \mu\right)\right|_{f}$ (if it is $2: 1$ ) so that they cannot be ramification points.

Thus we have seen that $\left.\left(\Phi_{n-1} \circ \mu\right)\right|_{f}$ is biholomorphic to the image, for a general fiber $f$ (of $\Phi_{n-1} \circ \mu$ ). Therefore $\Phi_{n-1} \circ \mu$, and therefore also $\Phi_{n-1}$, has to be birational, as required.

\section{ACKNOWLEDGMENT}

The author would like to thank the referee for a careful reading and a comment on the proof of Proposition 2.9 which makes it much clearer.

\section{REFERENCES}

[1] M. Atiyah, N. Hitchin, I. Singer, Self-duality in four-dimensional Riemannian geometry, Proc. Roy. Soc. London, Ser. A 362 (1978) 425-461. MR.506229 (80d:53023)

[2] N. Honda, Explicit construction of new Moishezon twistor spaces, J. Differential Geom. 82 (2009) 411-444. 
[3] N. Honda, Double solid twistor spaces: The case of arbitrary signature, Invent. Math. 174 (2008) 463-504. MR2453599

[4] N. Honda, A new series of compact minitwistor spaces and Moishezon twistor spaces over them, to appear in J. Reine Angew. Math., arXiv:0805.0042

[5] H. Kurke, Classification of twistor spaces with a pencil of surfaces of degree 1. I, Math. Nachr. 158 (1992) 67-85. MR:1235296 (94k:32051)

[6] C. LeBrun, Explicit self-dual metrics on $\mathbf{C P}^{2} \# \cdots \# \mathbf{C P}^{2}$, J. Differential Geom. 34 (1991) 223-253. MR 1114461 (92g:53040)

[7] H. Pedersen, Y. S. Poon, Self-duality and differentiable structures on the connected sum of complex projective planes, Proc. Amer. Math. Soc. 121 (1994) 859-864. MR 1195729 (94i:32049)

[8] Y. S. Poon, Compact self-dual manifolds of positive scalar curvature, J. Differential Geom. 24 (1986) 97-132. MR857378 (88b:32022)

[9] Y. S. Poon, On the algebraic structure of twistor spaces, J. Differential Geom. 36 (1992), 451-491. MR 1180390 (94a:32045)

Department of Mathematics, Tokyo Institute of Technology, O-okayama, Tokyo, JAPAN

E-mail address: honda@math.titech.ac.jp 